Relations industrielles

Industrial Relations

\title{
Unions and the Future, Industrial Relations Center, McGill University, Montreal, 1960.
}

\section{Gaston Cholette}

Volume 16, numéro 1, janvier 1961

URI : https://id.erudit.org/iderudit/1021896ar

DOI : https://doi.org/10.7202/1021896ar

Aller au sommaire du numéro

Éditeur(s)

Département des relations industrielles de l'Université Laval

ISSN

0034-379X (imprimé)

1703-8138 (numérique)

Découvrir la revue

Citer ce compte rendu

Cholette, G. (1961). Compte rendu de [Unions and the Future, Industrial Relations Center, McGill University, Montreal, 1960.] Relations industrielles / Industrial Relations, 16(1), 124-125. https://doi.org/10.7202/1021896ar

Tous droits réservés @ C Département des relations industrielles de l’Université Laval, 1961
Ce document est protégé par la loi sur le droit d'auteur. L'utilisation des services d'Érudit (y compris la reproduction) est assujettie à sa politique d'utilisation que vous pouvez consulter en ligne.

https://apropos.erudit.org/fr/usagers/politique-dutilisation/ 
fois, avec des confidences. Il ne faut pas $\mathrm{y}$ voir là des réflexions sentimentales, mais plutôt une auto-critique, fruit de l'expérience.

L'auteur s'en prend d'abord à la conception courante de l'entreprise selon laquelle elle est une propriété, propriété des patrons ou des capitalistes ou même de la nation. «La mentalité qui résulte de cette conception est fortement ancrée: ainsi, quand les travailleurs veulent se libérer de cette domination capitaliste, l'idée ne leur vient pas d'abord de faire reconnaître les droits du travail face à ceux du capital. Non, la conviction est établie que le maître de l'entreprise, c'est le propriétaire du capital. Alors on nationalise, c'est-à-dire qu'on substitue la nation aux capitalistes privés, sans que pour autant les droits des travailleurs soient fondamentalement modifiés... c'est par d'autres moyens qu'il faut tendre à s'affranchir de la trop exclusive tutelle du capital ».

L'auteur fait le procès de la participation aux bénéfices, de la participation au capital et des primes collectives de productivité. Et les deux points principaux de sa critique sont que l'on reste toujours sous la tutelle du capital et que l'on ne résout pas le problème de l'autofinancement. Ceux qui préconisent la participation aux bénéfices feraient bien de méditer ces pages. L'auteur commence par dire: «Personnellement, je parle d'une question que je connais, dont j'ai l'expérience pratique puisque dans l'entreprise que je dirige, j'ai instauré la participation du personnel aux bénéfices qui a été pratiquée de 1940 à $1947 . .$. Mais cette formule n'est pas bonne ». Et il développe trois raisons: a) l'ouvrier reste principalement un salarié; b) on laisse les capitalistes seuls s'enrichir de l'auto-financement; c) cette participation ne répond pas à l'aspiration des ouvriers pour lesquels le bénéfice est une notion lointaine, incontrôlable et qui leur semble impure. Il conclut de la façon suivante: «Je terminerai ces réflexions en disant que la participation, dont j'ai été un partisan et un pratiquant, m'apparaît, sur le tard, comme une forme attardée de paternalisme, et je crois que cette opinion deviendra commune quand on se sera rendu compte qu'elle n'est qu'un mauvais moyen de réaliser une fin qu'on se propose. Il en est d'elle comme de tous les moyens 《sociaux 》 de corriger les injustices et les dégâts provoqués par une mauvaise construction de départ: l'intention est généreuse, mais il vaut mieux se rendre compte que tout le soin et toutes les ressources consacrées à intégrer les travailleurs par ce moyen défectueux le seraient avec beaucoup plus d'efficacité dans une structure véritablement adaptée 》.

Il ne faudrait pas croire que l'auteur se contente de débâtir, de dissiper des illusions; la grande partie de son ouvrage est consacrée à élaborer et à justifier une véritable réforme de l'entreprise qui tient compte de toutes les réalités. L'entreprise est une entité distincte de la société de capitaux et des agents de production: elle est un centre d'échanges de services. Il faut distinguer entre la rémunération des serviteurs de l'entreprise et les acquêts de la communauté, lesquels sont nécessaires au maintien et au développement de l'entreprise et ne doivent pas être objet d'appropriation ni par les actionnaires ni par les travailleurs. L'auteur entre dans les détails des modalités techniques d'application et aborde les questions importantes d'autorité et de participation aux responsabilités, de rapport avec le syndicalisme et même de bilan véridique. En annexe, on trouve le texte de la première convention collective de rémunération d'entreprise de l'en. treprise qu'il dirige.

Confidences d'un patron est un ouvrage sérieux qui va au fond de la question de la réforme de l'entreprise. Les vrais problèmes sont posés tant sur le plan doctrinal que sur le plan pratique. Tous ceux qu'intéresse cette question difficile tireront grand profit à le lire.

\section{GÉrard Dion}

Unions and the Future, Industrial Relations Center, McGill University, Montreal, 1960.

Il s'agit du rapport du lle congrès organisé par le Centre des relations industrielles de l'Université McGill et qui portait sur les syndicats ouvriers face à l'avenir.

Le premier conférencier, R.A. Lester, fait une revue de la dernière décade. II constate que la phase militante du mouvement ouvrier américain est révolue et que la maturation du syndicalisme ouvrier se traduit par un certain embourgeoisement. 
Le deuxième conférencier, Sylvio Ostey, traite de quelques aspects de la structure des salaires au Canada et des conséquences qui en découlent pour le mouvement ouvrier. L'auteur fournit des renseignements intéressants sur les différences de salaires par occupations, par branches d'activité et par régions, en analysant et en commentant leurs variations au cours d'une quinzaine d'années.

Une troisième étude importante, par Archibald Cox, porte sur l'intervention de l'Etat dans la régie interne des syndicats ouvriers aux Etats-Unis. L'auteur fait ressortir les implications du LaborManagement Reporting and Disclosure Act en retraçant brièvement les principales tendances de l'opinion publique et du législateur à l'égard du mouvement ouvrier au cours des vingt dernières années.

Enfin, le rapport contient une dernière conférence sur la juridiction fédérale dans le domaine des relations industrielles. L'auteur, F.R. Scott, est d'avis que le Parlement ne détient pas suffisamment de pouvoirs et qu'il faudrait lui confier la tâche de contrôler les négociations qui, en fait, se font sur le plan national.

Le rapport se termine par une discussion à laquelle ont participé MM. K.G.K. Baker, Eugène Forsey, D.G. Pyle et Leo Roback.

\section{G. Cholette}

Pourquoi les travailleurs abandonnent la terre: Etude comparative, Bureau international du Travail, Genève, 1960, 267 pp., \$2.25.

Comme toutes les études du Bureau international du Travail, cette monographie réussit à présenter une synthèse très valable d'un problème complexe. Les deux questions principales que traite cette étude sont 1) quelle est l'ampleur du mouvement de la migration des travailleurs ruraux vers la ville, 2) pourquoi cette migration a-t-elle lieu? De façon moins systématique, le problème des conséquences socio-économiques de cette migration est aussi traité.

Comme les auteurs veulent donner une vue synthétique du problème dans l'ensemble des pays du globe, ils ont divisé en trois catégories les états selon leur degré de développement économique. On pourra ainsi analyser les situations et les problèmes particuliers des états économiquement évolués, des états dont les campagnes sont surpeuplées et dont le rythme de croissance est lent, des états dont le développement s'effectue à un rythme accéléré. Cette division permet non seulement de montrer les différences dans la nature et la signification de la migration hors des campagnes mais permet aussi, en conclusion, de suggérer des politiques nationales qui sont mieux adaptées aux besoins réels des différents états.

Ce volume est donc d'un très grand intérêt aussi bien pour le chercheur qui y trouve à la fois des données bien colligées et des hypothèses intéressantes que pour l'homme d'action qui $y$ trouve l'exposé des principaux faits dont il doit tenir compte et des suggestions pratiques pertinentes.

Il faut, en particulier, féliciter les auteurs de leur excellente utilisation des statistiques officielles des différents pays étudiés. Quand on connait la très grande diversité des techniques et des défnitions employées dans les recensements, une étude comparative basée sur ces sources apparaît une aventure très périlleuse. Les auteurs ont su avertir le lecteur des dangers d'une comparaison trop rigide entre les pays. Ils ont quand même su utiliser au maximum les données dont ils disposaient. Le schéma global qu'ils tracent garde sa valeur entière en dépit de l'imprécision des sources.

Si cet effort de comparaison a été fructueux, c'est sans aucun doute dû en grande partie au cadre de référence utilisé dans la monographie. Les auteurs ne cherchent pas seulement à présenter des faits mais aussi, sinon plus, à vérifier un certain nombre d'hypothèses cohérentes. Ces hypothèses, comme l'indique déjà le choix des catégories employées pour classifier les états, mettent en relation l'émigration rurale et le développement économique. Deux facteurs sont considérés comme importants pour expliquer l'abandon de la terre: 1) les bas revenus agricoles relativement aux revenus des autres secteurs économiques (facteur de refoulement); 2) possibilités d'emplois en dehors de l'agriculture (facteur d'appel). Dans les pays économiquement évolués et dans les pays en voie de développement rapide les deux facteurs vont jouer à la fois. L'émigration rurale sera ainsi un élément actif du progrès 